\title{
Nitrogen Hydrate Cage Occupancy and Bulk Modulus Inferred from DFT-derived Cell Parameters
}

C. Métais ${ }^{\mathrm{a}, \mathrm{b}, \mathrm{c}}$, C. Petuya ${ }^{\mathrm{b}}$, S. Espert ${ }^{\mathrm{b}, \mathrm{d}}$, J. Ollivierc ${ }^{\mathrm{c}}$, L. Martin-Gondre, ${ }^{\mathrm{a},}$, A. Desmedt ${ }^{\mathrm{b}, *}$

a) Institut UTINAM, UMR 6213 CNRS, Université de Bourgogne - Franche-Comté, Besançon, France

b) Groupe Spectroscopie Moléculaire, Institut des Sciences Moléculaires (ISM), UMR 5255 CNRS, Université de Bordeaux, France

c) Groupe Spectroscopie, Institut Laue Langevin (ILL), Grenoble, France

d) Donostia International Physics Center (DIPC), Paseo Manuel de Lardizabal 4, 20018 San Sebastián, Spain

*Corresponding authors : arnaud.desmedt@u-bordeaux.fr ; ludovic.martin@univ-fcomte.fr

\section{Content}

- Comparison of lattice parameter dependence of the DFT potential energy for the PBE and vdW-DF functionals

- Non-bonding, guest-host and host-host potential energies calculated with the vdW-DF functional

- Non-bonding, guest-host and host-host potential energies calculated with the PBE functional

- Reference 


\section{Comparison of lattice parameter dependence of the DFT potential energy for the PBE and}

\section{$\underline{\text { vdW-DF functionals }}$}
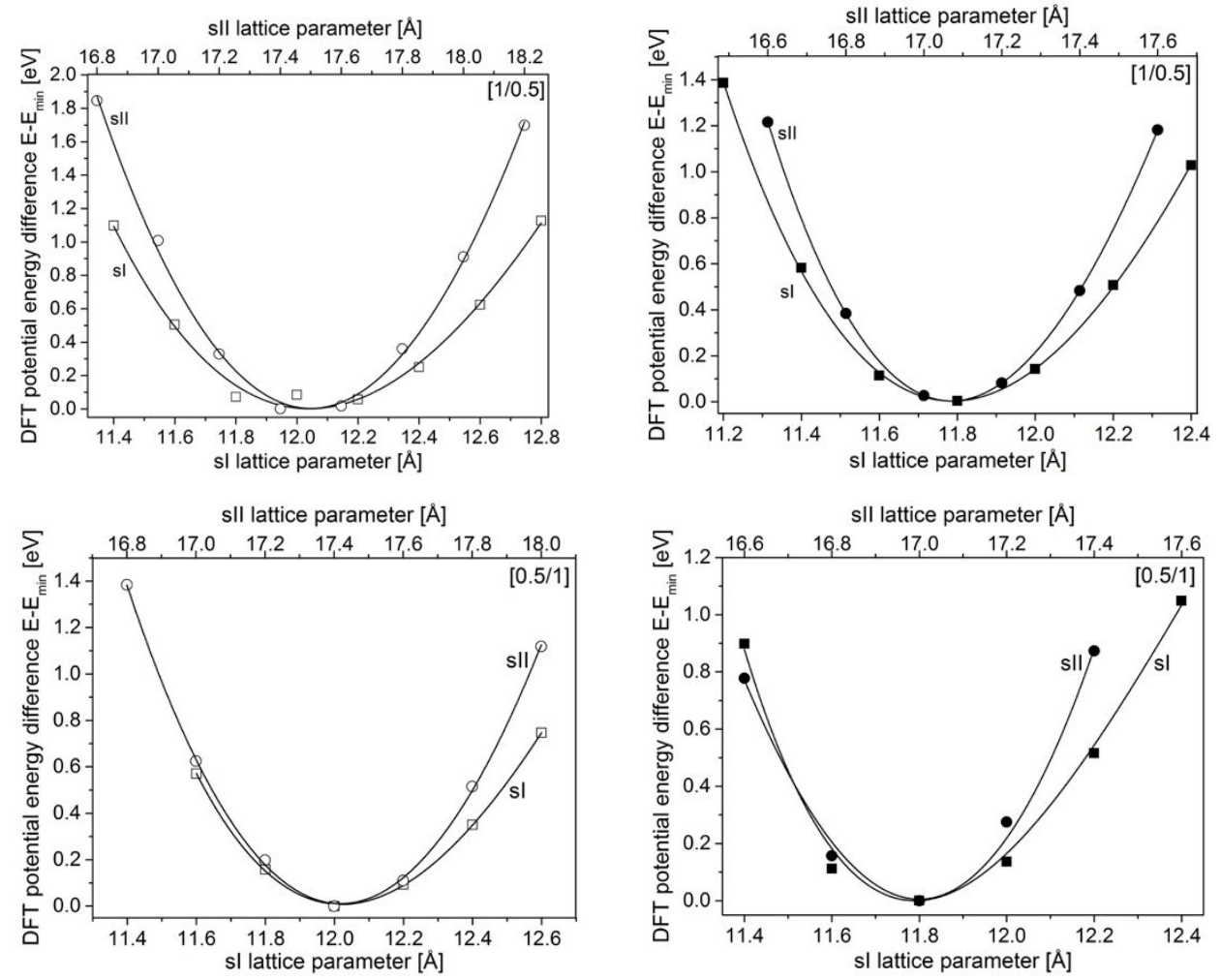

Figure S1: DFT potential energy difference E-Emin, where $E_{\min }$ is the minimal potential energy, as a function of the lattice parameter obtained from DFT calculations made on systems with all SCS and half LCS simply filled (top, [1/0.5]) and on systems with half SCS and all LCs simply filled (bottom, [0.5/1]). Results obtained with the vdW-DF and PBE functionals are represented by empty and solid symbols, respectively. SI and sll are represented by squares and circles, respectively. The continuous lines represent the fitted curves using the Birch-Murnaghan equation of state [Birch 1947]. 


\section{Non-bonding, guest-host and host-host potential energies calculated with the vdW-DF}

\section{functional}

Table S1: Potential energies per molecule (eV/molecule) calculated with vdW-DF functional for the sl and sll nitrogen hydrate. $E^{N B}$ is the nonbonding energy, $E^{H H}$ is the host-host energy and $E^{G H}$ is the guest-host energy. All the performed simulations results are presented in this table.

Clathrate structure I

\begin{tabular}{|c|c|c|c|c|c|c|}
\hline $\begin{array}{l}\text { Labeling } \\
{\left[\boldsymbol{\theta}_{\mathrm{SC}} / \boldsymbol{\theta}_{\mathrm{LC}}\right]}\end{array}$ & $\mathrm{E}^{\mathrm{NB}}$ & $\mathrm{E}^{\mathrm{HH}}$ & $\mathrm{E}^{\mathrm{GH}}$ & $\mathrm{E}^{\mathrm{NB}}$ & $\mathrm{E}^{\mathrm{HH}}$ & $\mathrm{E}^{\mathrm{GH}}$ \\
\hline$[0 / 1]$ & -0.52 & -0.536 & -0.396 & -0.531 & -0.542 & -0.35 \\
\hline$[1 / 0]$ & -0.538 & -0.546 & -0.371 & -0.514 & -0.529 & -0.38 \\
\hline$[0.5 / 1]$ & -0.516 & -0.536 & -0.385 & -0.52 & -0.537 & -0.373 \\
\hline$[1 / 0.5]$ & -0.529 & -0.545 & -0.381 & -0.516 & -0.537 & -0.379 \\
\hline$[1 / 1]$ & -0.515 & -0.535 & -0.399 & -0.515 & -0.539 & -0.376 \\
\hline$[1 / 1.125]$ & & & & -0.513 & -0.539 & -0.372 \\
\hline$[1 / 1.17]$ & -0.507 & -0.536 & -0.359 & & & \\
\hline$[1 / 1.25]$ & & & & -0.503 & -0.529 & -0.368 \\
\hline [1/1.33] & -0.499 & -0.535 & -0.331 & & & \\
\hline$[1 / 1.375]$ & & & & -0.502 & -0.528 & -0.366 \\
\hline$[1 / 1.5]$ & -0.498 & -0.54 & -0.321 & -0.503 & -0.533 & -0.359 \\
\hline$[1 / 1.625]$ & & & & -0.498 & -0.528 & -0.355 \\
\hline$[1 / 1.67]$ & -0.491 & -0.54 & -0.304 & & & \\
\hline$[1 / 1.75]$ & & & & -0.506 & -0.539 & -0.355 \\
\hline$[1 / 1.83]$ & -0.485 & -0.539 & -0.293 & & & \\
\hline$[1 / 1.875]$ & & & & -0.503 & -0.537 & -0.356 \\
\hline$[1 / 2]$ & -0.472 & -0.529 & -0.286 & -0.508 & -0.545 & -0.352 \\
\hline
\end{tabular}




\section{Non-bonding, guest-host and host-host potential energies calculated with the PBE}

\section{functional}

Table S2: Potential energies per molecule (eV/molecule) calculated with PBE functional for the sl and sll nitrogen hydrate. $E^{N B}$ is the nonbonding energy, $E^{H H}$ is the host-host energy and $E^{G H}$ is the guest-host energy. All the performed simulations results are presented in this table.

Clathrate structure I

\begin{tabular}{|c|c|c|c|c|c|c|}
\hline $\begin{array}{l}\text { Labeling } \\
{\left[\boldsymbol{\theta}_{\mathrm{SC}} / \boldsymbol{\theta}_{\mathrm{LC}}\right]}\end{array}$ & $\mathrm{E}^{\mathrm{NB}}$ & $\mathrm{E}^{\mathrm{HH}}$ & $\mathrm{E}^{\mathrm{GH}}$ & $\mathrm{E}^{\mathrm{NB}}$ & $\mathrm{E}^{\mathrm{HH}}$ & $\mathrm{E}^{\mathrm{GH}}$ \\
\hline$[0 / 1]$ & -0.549 & -0.615 & -0.045 & -0.588 & -0.619 & -0.055 \\
\hline$[1 / 0]$ & -0.588 & -0.615 & 0.03 & -0.546 & -0.612 & 0.017 \\
\hline$[0.5 / 1]$ & -0.538 & -0.614 & -0.038 & -0.557 & -0.62 & -0.024 \\
\hline$[1 / 0.5]$ & -0.555 & -0.615 & -0.003 & -0.528 & -0.605 & -0.01 \\
\hline$[1 / 1]$ & -0.528 & -0.614 & -0.031 & $-0,516$ & $-0,604$ & $-0,019$ \\
\hline$[1 / 1.125]$ & & & & -0.508 & -0.599 & -0.016 \\
\hline$[1 / 1.17]$ & -0.494 & -0.586 & -0.024 & & & \\
\hline$[1 / 1.25]$ & & & & -0.509 & -0.606 & -0.002 \\
\hline [1/1.33] & -0.482 & -0.591 & 0.018 & & & \\
\hline$[1 / 1.375]$ & & & & -0.506 & -0.606 & -0.003 \\
\hline$[1 / 1.5]$ & -0.464 & -0.579 & 0.017 & -0.506 & -0.611 & 0.005 \\
\hline$[1 / 1.625]$ & & & & -0.488 & -0.591 & -0.004 \\
\hline [1/1.67] & -0.459 & -0.585 & 0.026 & & & \\
\hline [1/1.75] & & & & -0.496 & -0.606 & 0.003 \\
\hline$[1 / 1.83]$ & -0.443 & -0.582 & 0.051 & & & \\
\hline$[1 / 1.875]$ & & & & -0.492 & -0.606 & 0.011 \\
\hline$[1 / 2]$ & -0.43 & -0.569 & 0.028 & -0.481 & -0.597 & 0.009 \\
\hline
\end{tabular}




\section{Reference}

[Birch 1947] Birch, F. Finite Elastic Strain of Cubic Crystals", Phys. Rev. 1947, 71, 809-824. 